\title{
Analyse du Contexte Politique et Socio-Economiques et des Differences Qu'il Peut Apporter au Marketing Politique en Tant Que Concept Theorique : Maroc - Legislatives 2016
}

\author{
Pr. Makry Hajar, Docteur en Sciences de Gestion \\ Professeur de l'Enseignement Supérieur Assistant \\ Faculté Polydisciplinaire de Sidi Bennour, \\ Université Chouaib Doukkali, Morocco
}

Doi:10.19044/esj.2021.v17n23p249

Submitted: 14 June 2021

Accepted: 14 July 2021

Published: 31 July 2021
Copyright 2021 Author(s)

Under Creative Commons BY-NC-ND

4.0 OPEN ACCESS

Cite As:

Hajar M. (2021). Analyse du Contexte Politique et Socio-Economiques et des Differences Qu'il Peut Apporter au Marketing Politique en Tant Que Concept Theorique : Maroc Legislatives 2016. European Scientific Journal, ESJ, 17(23), 249.

https://doi.org/10.19044/esj.2021.v17n23p249

\section{Résumé}

En dépit de l'attention qu'a reçue le marketing politique et des progrès conceptuels et empiriques qui ont été accomplis, les chercheurs reconnaissent souvent les différences et les difficultés de conceptualisation et d'opérationnalisation de ce construit. L'action politique est un événement contextuel et est le résultat de nombreuses influences. À travers des recherches documentaires et des entretiens semi-directifs avec des observateurs-experts (journalistes et académiciens) en matière de la chose politique, cette contribution a essayé de voir si le marketing politique présentedes caractéristiques similaires dans des contextes politique et socioéconomique différents. Nous soutenons l'idée d'une prise en considération plus explicite des facteurs contextuels dans la dynamique des actions marketing des partis politiques au Maroc. La présente contribution est le résultat d'une étude de contextualisation de notre travail de recherche sur le marketing politique et la performance électorale. Un contexte délimité en l'année législative 2016.

Mots-clés: Contexte, législatives 2016, Marketing Politique, Marketing Stratégique Maroc 


\title{
Analysis of Political and Socio-Economic Context and the Influence it Can Bring to Political Marketing as a Theoretical Concept: Morocco - Parliamentary Elections 2016
}

\author{
Dr. Makry Hajar, Doctor of Management Sciences \\ Assistant Higher Education Professor \\ Sidi Bennour Polydisciplinary Faculty, \\ Chouaib Doukkali University, Morocco
}

\begin{abstract}
Despite the attention that political marketing has received and the conceptual and empirical advances that have been made, researchers still face difficulties in conceptualizing and operationalizing the concept. Political action is a contextual event and is the result of many influences. Through a documentary research and semi-structured interviews with observers and experts (journalists and academics) in politics and marketing, this paper focuses on ascertaining if political marketing presents similar characteristics in different political and socio-economical contexts. The study also focused on a more explicit consideration of contextual factors in the marketing actions dynamics of political parties in Morocco. This contribution is the output of a contextualization study of our research on political marketing and electoral performance.
\end{abstract}

Keywords : Context, 2016 legislative elections, Morocco, Political Marketing, Strategic Marketing

\section{Introduction}

En dépit de l'attention qu'a reçue le marketing politique et des progrès conceptuels et empiriques qui ont été accomplis, les chercheurs reconnaissent souvent les différences et les difficultés de conceptualisation et d'opérationnalisation de ce construit.

Sur le plan pratique, l'ouverture du champ politique marocain et la montée du digital semblent bouleverser la communication dans le Royaume. Jusqu'aux années 2000, la communication politique était plutôt verticale, de haut en bas, et à sens unique. Cela signifie que la communication était émise, sous forme de communiqués ou de diffusion d'informations, sans tenir compte des spécificités des cibles, que ce soit au niveau de la forme ou du fond.

L'action politique, en tant que processus complexe, ne peut pas s'expliquer sans la prise en compte des interactions entre l'acteur politique et son environnement. L'action politique est un événement contextuel et est le 
résultat de nombreuses influences. Ainsi une question importante peut être résumée par la formulation suivante : le marketing politique présente-t-il des caractéristiques similaires dans des contextes politique et socio-économique différents ?

Cette notion de « contexte », étroitement liée au paradigme compréhensif, a reçu beaucoup d'attention en sciences humaines et sociales. Toutefois, l'analyse qualitative et contextuelle des problématiques politiques semble être occultée par le quantitatif confirmatoire qui domine largement la recherche en sciences sociales.

Nous soutenons l'idée d'une prise en considération plus explicite des facteurs contextuels dans la dynamique des actions marketing des partis politiques au Maroc. La présente contribution explique pourquoi et en quoi les questions de contexte peuvent être pertinentes dans une recherche qualitative pour consolider le cadre d'analyse et d'interprétation des résultats.

Dans ce qui suit, nous présentons la démarche et les principaux résultats d'une étude de contextualisation, conduite dans le cadre de notre recherche, sur les pratiques du marketing des partis politiques au Maroc. L'objectif est donc de présenter les différents aspects qui caractérisent la fondation de notre design de recherche par l'intégration des considérations, non seulement épistémologiques, mais aussi contextuels qui justifient notre cadre théoricoconceptuel et nos choix méthodologiques.

\section{De l'importance épistémologique du contexte}

La diversité des paradigmes épistémologiques est un élément essentiel pour expliquer le caractère fragmenté et peu cumulatif des connaissances scientifiques dans les sciences sociales. Buchanan et Bryman (2007), considèrent que les recherches qualitatives et interprétatives semblent de plus en plus caractérisées par une «inventivité » méthodologique en ce qui concerne les démarches d'investigation empirique, de conceptualisation et de construction théorique.

L'utilisation des méthodes qualitatives assoit sa légitimité de la définition de l'objet de la recherche, en plus de la faculté que se donne le chercheur de laisser émerger ou advenir des significations autres que celles liées directement au focus. Or, les choix conceptuels et méthodologiques ne peuvent pas être présumés par le seul choix épistémologique (Guba \& Lincoln, 1994), ou seulement par des considérations techniques et théoriques liées à l'objet de recherche (Buchanan \& Bryman, 2007). Ces choix doivent être inscrits dans un système global de recherche où plusieurs facteurs (théorique, épistémologique, organisationnel, historique, éthique et personnel) sont combinés d'une manière cohérente dans la poursuite des objectifs particuliers dans un contexte donné. Dans ce sens, la démarche de contextualisation aide 
à révéler l'influence du contexte sur la construction de l'objet de la recherche et sur la méthode d'approche.

Le concept de contextualisation s'inscrit dans le courant théorique de l'approche systémique ; plus précisément dans le prolongement du modèle des interactions en contexte (Bru, 1991 cité par Marcel, 2002). Elle insiste sur la nécessité d'étudier les comportements des acteurs dans le contexte de leur actualisation. Une définition générique du contexte est fournie par (Dey, 2001), qui le définit comme " toute information susceptible d'être utilisée pour caractériser la situation d'un phénomène ». Il désigne alors les circonstances accompagnant un phénomène et sa mise en relation avec des éléments constitutifs de son environnement. Plus précisément, le contexte traduit "l'ensemble des circonstances qui accompagnent un évènement, il devient un élément incontournable des recherches de type qualitatif qui insistent sur le fait que les sujets ne sont pas réduits à des variables, mais sont considérés comme un tout » (Pourtois \& Desmet, 2004).

La contextualisation suppose que le travail du chercheur se situe sur le terrain réel des acteurs (Bronfenbrenner, 1977 cité par Parrini-Alemanno, 2007), qu'elle s'associe une validité de contexte (caractéristiques du contexte social et culturel des acteurs), et une validité phénoménologique (signification de la situation pour les acteurs).

Permettant de cadrer et de pré-objectiver les analyses du chercheur, la contextualisation devient alors un élément, à la fois épistémique et méthodologique, fondamental doté d'une importance particulière dans le paradigme compréhensif et les recherches de type qualitatif (Pourtois \& Desmet, 2004).

Le contexte organisationnel renvoie méthodologiquement, à la recherche du cadre interprétatif commun à l'ensemble concerné par la recherche. Le contexte phénoménologique (Parrini-Alemanno, 2007) met la lumière sur la perception qu'ont les acteurs du milieu et de ses éléments. Dans ce sens, la pluralité des facettes du contexte offre une perspective méthodologique de traitement de l'information utile pour la compréhension de la réalité de la situation à étudier (Parrini-Alemanno, 2007). Dès lors, la signification et la contextualisation deviennent deux facettes du même processus témoignant de l'effort permanent des acteurs pour comprendre leur environnement, notamment dans les organisations.

Par la mise en exergue des dynamiques contextuelles en jeu, la démarche de contextualisation permet de (re)construire l'objet de la recherche et sa méthode d'approche et ainsi de favoriser l'émergence et le recadrage d'une situation pertinente pour le problème. 


\section{Méthode de conduite de la démarche de contextualisation}

Les processus sociaux sont fondamentalement dynamiques et ne sont pas directement observables. Nous sommes donc contraints de recourir à de multiples collectes d'informations pour étudier ces processus. L'étude de contextualisation a été alors mise en œuvre à travers des recherches documentaires et des entretiens semi-directifs avec des observateurs-experts (journalistes et académiciens) en matière de la chose politique. Nous avons utilisé un guide d'entretien assez détaillé à travers lequel nous souhaitions cadrer leurs perceptions des leviers des orientations marketing des partis politiques marocains lors des campagnes électorales.

La démarche a été complétée par une analyse documentaire qui a été basée essentiellement sur les rapports publiés par des instances nationales et internationales. Cinq entretiens semi-directifs ont été menés avec différents journalistes et académiciens (cf. Tableau 1).

Il reste à répondre à la question difficile du nombre d'interviewés à retenir. Pour Hlady Rispal (2002), le choix du nombre d'interviewés dépend des concepts de saturation théorique, et de généralité de l'étendue.

La saturation théorique : est le moment à partir duquel l'apprentissage devient minime, Strauss et Corbin (1998) estiment que la saturation théorique est atteinte quand rien de consistant ou de nouveau n'émerge du terrain. Guillemette (2006) explique que le plus important est l'émergent- fit, qui consiste en une cohérence entre le jugement du chercheur et le fait que les données qui émergent du terrain ne font pas vraiment avancer l'analyse.

La généralité de l'étendue : ou la réplication de l'étendue. Yin (1994) explique que le nombre de cas dépend du degré de certitude souhaité par le chercheur. Il s'agit de répliquer le cas jusqu'à ce qu'aucun résultat nouveau n'apparaisse après l'analyse.

De manière générale, le choix du nombre des interviewés dépend des moyens du chercheur ainsi que de l'objet de la recherche, la saturation théorique se combine avec des raisons très pragmatiques de temps et de moyens (Strauss \& Corbin, 2004). 
Tableau 1. Liste des interviewés

Interview 1 Blogueur/Journaliste Hôtel Bistrot du Piétri/ Face à Face/ Rabat MP\# OBSERVATEUR Rabat

\begin{tabular}{|c|c|c|c|c|c|}
\hline $\begin{array}{l}\text { Interview } 2 \\
\text { OBSERVATEUR }\end{array}$ & $\begin{array}{l}\text { Rédacteur En chef } \\
\text { d'un journal Partisan }\end{array}$ & $\begin{array}{l}\text { Au siège du Journal/ } \\
\text { Casablanca }\end{array}$ & $\begin{array}{l}\text { Face à } \\
\text { Casablanca }\end{array}$ & Face/ & MP\#2 \\
\hline $\begin{array}{l}\text { Interview } \\
\text { OBSERVATEUR }\end{array}$ & $\begin{array}{l}\text { Professeur Chercheur } \\
\text { (sciences juridique) }\end{array}$ & Café littéraire / Marrakech & $\begin{array}{l}\text { Face à } \\
\text { Marrakech }\end{array}$ & Face & MP\#3 \\
\hline $\begin{array}{l}\text { Interview } \\
\text { OBSERVATEUR }\end{array}$ & Journaliste-Hespress & $\begin{array}{l}\text { Marrakech-à la marge du } \\
\text { forum des nations unies des } \\
\text { services public }\end{array}$ & $\begin{array}{l}\text { Face à } \\
\text { Marrakech }\end{array}$ & Facel & MP\#4 \\
\hline $\begin{array}{l}\text { Interview } 5 \\
\text { OBSERVATEUR }\end{array}$ & $\begin{array}{l}\text { Consultant } \\
\text { marketing }\end{array}$ & Café Starbucks / Casablanca & $\begin{array}{l}\text { Face à } \\
\text { Casablanca }\end{array}$ & Face/ & MP\#5 \\
\hline
\end{tabular}

\section{Déroulement de la recherche}

C'est la première phase du terrain de la recherche, elle a débuté avec des entretiens d'observateurs du début avril à fin mai 2018. L'objectif de ces entretiens, et en plus de nous éclairer sur le contexte d'application du marketing politique, est de nous orienter sur la manière dont les partis s'organisent, en fonctions de leurs contextes respectifs, et de nous éclairer sur les performances comparées de ces partis.

Après avoir analysé et interprété les entretiens d'observateurs, nous avons arrêté la structure finale des entretiens individuels : 


\section{Contexte général des partis politiques}

1. Pouvez-vous parler de votre vision générale de la scène politique au Maroc ?

$\rightarrow$ Quelle est la raison de la baisse des partisans du parti ?

$\rightarrow$ En ce qui concerne les partisans qui participent au travail politique, est-ce que les partis politiques possèdent, selon vous, une stratégie pour les attirer? Y'a-t-il une catégorie spécifique ciblée ? Ou les partis orientent leurs discours à toutes les catégories de la société ?

2. Que pensez-vous des programmes électoraux de 2016 ?

$\rightarrow$ Est-ce suffisant?

$\rightarrow$ Est-ce que les procédures suivies par les partis politiques dans la sélection des candidats les aident à choisir des candidats ayant de la popularité auprès des citoyens ?

\section{Les moyens des partis politiques}

$\rightarrow$ Les budgets alloués par les partis politiques dans la communication et le marketing politique sont-ils adéquats?

\section{Les élections de 2016}

1. Quel est votre commentaire sur les élections de 2016 ?

$\rightarrow$ Est-ce que les partis politiques, ont-ils utilisé professionnellement les réseaux sociaux et les sites web dans la campagne de 2016 ?

2. Selon vous, quels sont les obstacles qui empêchent les partis politiques de pratiquer un marketing politique professionnel ? Est-ce le manque de compétence ? le manque de budget? Est-ce l'héritage conservateur qui empêche d'acquérir des nouvelles technologies?

\section{Regards d'ordre général}

1. Si nous comparons le Maroc avec les pays développés tels que les États-Unis d'Amérique et les pays européens?

2. Selon vous, quel est le parti politique le plus présent sur les réseaux sociaux?

3. Selon votre propre expérience, comment définissez-vous le marketing politique ? est-il connecté avec les campagnes électorales ?

\section{Le marketing politique au Maroc : des actions diversifiées et un caractère conjoncturel}

\subsection{Particularité du marketing politique au Maroc}

Indissociable des techniques empruntées du marketing des entreprises, le marketing politique se définit comme le processus par lequel les candidats et les idées politiques sont dirigés vers les électeurs afin de satisfaire leurs besoins politiques et ainsi obtenir leur soutien pour le candidat et les idées en question (Shama 1975 cité par Makry 2020)

L'application du marketing traditionnel à la politique était justifiée par un certain nombre de similitudes des similarités de concepts (par exemple, consommateurs, segmentation du marché, mix marketing, image, fidélité à la marque, concept de produit et positionnement) et des similarités d'outils (par exemple, étude de marché, communication et publicité) (Egan, 1999 ; Kotler, 1975). 
Les pratiques du marketing politique électoral des partis politiques au Maroc ont commencé à évoluer avec l'adoption du nouveau mode de scrutin basé sur les listes en 2002. En effet, après une ère de scrutin individuel basée sur une relation directe avec les citoyens et marquée par des campagnes de communication directe (porte-à-porte, affiches, journaux, etc.), c'est l'époque de l'usage de moyens sophistiqués de communication politique axés sur la variété des moyens de diffusion massive, principalement traversés par la télévision, l'Internet et la téléphonie mobile.

L'observation des campagnes électorales des différents partis lors des élections législatives 2016 montre que le marketing politique a fait son entrée sur la scène marocaine, pour le moins chez certains partis.

D'une manière générale, les politiciens utilisent divers moyens pour faire adhérer les citoyens à leurs programmes politiques ainsi qu'à leurs actions. Toutefois, le recours aux techniques marketing politique reste un phénomène relativement récent.

La communication politique électorale des partis n'a pas encore atteint le degré de professionnalisation requis. De ce fait, elle est plus proche de la propagande que du marketing politique qui met en avant la primauté de la demande (besoins des électeurs potentiels).

L'introduction des techniques de marketing requiert l'adoption d'une démarche globale de conception, de rationalisation et d'accomplissement de la communication politique moderne.

Toutefois, ces premiers constats doivent être nuancés par la prise en compte de certaines particularités juridico-institutionnelles, culturelle et historiques du contexte marocain qui ne permettent pas encore la réalisation des campagnes électorales marketées dans les conditions idéales définit par la science.

\subsection{L'impact limité des pratiques du Marketing politique : le poids des freins socio-culturels}

Pour comprendre le caractère conjoncturel de la communication de la plupart des partis, il faut tenir compte du contexte sociopolitique dans lequel elle est déployée. Ainsi, ce contexte est marqué par un bon nombre de facteurs, dont principalement le monopole qu'exerce l'Etat sur les médias officiels marocains, que la grande limitation des moyens financiers des partis politiques marocains, ou alors le faible intérêt que porte l'élite partisane à la professionnalisation de la communication.

La voix de l'électorat n'était pas intégrée en amont. Aujourd'hui, la voix de l'électorat se ressent avec force, et la multiplication des enquêtes du comportement électoral et des intentions de vote témoigne de cette importance. 
Les élections ne sont plus la seule forme d'engagement politique chez les jeunes, et on assiste à une quasi-dépolitisation du citoyen aujourd'hui, à cause de l'érosion de la crédibilité des partis politiques et des institutions élues, ce qui donne au citoyen un jugement préalable sur la futilité de la pratique politique.

Deux facteurs principaux peuvent expliquer cette méfiance du citoyen marocain selon notre interviewé MP\#3 :

D'abord, les projets des partis politiques sont très identiques au niveau de la conformité des programmes et des discours, aussi bien dans les rencontres rhétoriques, dans le parlement par le biais des questions verbales, ou même dans les programmes de télévision. Auparavant, chaque parti possédait un langage spécifique qui le distingue des autres partis, et qui reflétait la vision du parti et son orientation politique, mais aujourd'hui le langage est le même et il n'y a plus de projets spéciaux. Le deuxième facteur est de nature quantitative et concerne le nombre des voix à obtenir. Les résultats ne sont plus falsifiés au niveau des urnes, mais les fraudes ont lieu en dehors de ces urnes, en achetant des votes et en faisant d'autres pratiques non démocratiques. MP\#3.

Toutefois la situation diffère d'un parti à l'autre, on trouvera, à titre d'exemple, que le nombre des partisans du Parti de la Justice et du Développement (PJD) a augmenté, tandis que ceux de l'Union Socialiste des Forces Populaires (USFP) a diminué.

Selon nos observateurs, cette situation peut s'expliquer par le fait que Le PJD a bénéficié de toute cette situation caractérisée par le déclin de la démocratie interne. C'est un parti qui a montré des modèles très distincts dans sa démocratie interne et il était présent dans tous les quartiers, villes et villages du Maroc. Bien qu'il y eût un déclin dans les organisations parallèles, le PJD a étendu les ponts de coopération avec plusieurs de ses alliés, même s'ils apparaissent indépendants ; ce qui l'a encore aidé c'est l'usage d'un discours religieux dans une communauté religieuse conservatrice qui n'a jamais expérimenté le travail politique d'un parti islamique.

Donc au sein de cette ambiance caractérisée par le déclin de l'action politique, le PJD a réussi à réaliser du progrès, au contraire de la majorité des partis politiques marocains.

Bien que la communication politique de la plupart des partis politiques marocains ait connu une sophistication de contenu et de forme, plusieurs problèmes de taille empêchent sa perception positive et son efficacité. Plusieurs facteurs peuvent expliquer ce faible impact du marketing politique au Maroc sur l'électorat, en l'occurrence le mode de scrutin proportionnel, la multitude des partis (plus de 30 partis au total à la veille des élections 2016), le clanisme (surtout dans le monde rural), le manque d'implication politique 
et le manque de confiance des électeurs, l'analphabétisme, le manque de compétence des cadres des partis, l'héritage conservateur, etc.

Les partis politiques sont de plus en plus en lutte pour la préservation et la durabilité des sièges de leurs membres. On trouve aussi le problème de l'inefficacité de la communication traditionnelle, et surtout l'absence d'un contenu intéressant.

Étant donné les mécanismes de la loi électorale, il s'avère extrêmement difficile pour tout parti de réaliser une nette majorité au Parlement. De plus, il peut y avoir un important décalage entre le nombre de votes populaires recueillis par les différents partis et leur représentation au sein du Parlement élu. MP\#1

La pratique marketing politique caractérise, normalement, un environnement démocratique puisqu'il met au centre de son attention les électeurs et non les candidats. Toutefois, les observateurs restent peu optimistes quant à la capacité des partis politiques marocains à mettre en œuvre une véritable politique marketing électorale, et ce pour plusieurs raisons. Notre interviewé précise :

Il faut savoir deux choses, la première c'est que certains partis politiques n'ont toujours pas saisi l'importance de la communication et du marketing politique, et la seconde c'est que certains partis sont conscients de l'importance de la communication et du marketing politique, mais ne l'utilisent pas, et ne cherchent pas le faire. En effet, ils n'ont même pas de pages Facebook officielles. Ceci est dû à plusieurs raisons, dont la plus importante est que ceux qui dirigent et sélectionnent et fixent les budgets n'ont pas encore saisi l'importance de la révolution numérique. MP\#4.

Pourtant, les observateurs citent encore l'exemple du PJD pour démontrer l'importance des pratiques du marketing politique dans l'amélioration de l'image du parti malgré les failles de gestion gouvernementales.

La règle générale dit que chaque parti qui exerce le pouvoir voit sa base électorale se réduire. Mais cette règle n'a pas été appliquée dans le cas du PJD. Ceci a constitué la première et la plus grande remarque. La deuxième remarque est que, en 2016, il a été constaté que les partis politiques vantent leurs expériences dans les espaces virtuels, ce qui pousse tout observateur à se croire en face d'un véritable pouvoir électoral, mais les résultats ne sont pas aussi importants pour être célébrés. L'expérience de la Fédération de la Gauche Démocratique est le meilleur exemple. Ceux qui ont suivi ce parti sur Facebook et sur Twitter ont cru, en se basant sur les interactions positives et les réactions positives obtenues par le 
peuple à leur comportement et à leurs choix, que ce parti serait la deuxième force politique après le PJD, MP\#2.

D’une manière générale, la pratique du marketing politique au Maroc reste tributaire de son contexte d'application. Le scepticisme des citoyens quant à la gestion de la chose politique au Maroc reste le constat dominant. Toutefois, on assiste à une faible différenciation créée par le PJD, et qui est majoritairement due à son idéologie qui trouve bon écho auprès de son environnement.

\section{Crise de projets et de vision stratégique des partis politiques 5.1. Déficit de différenciation des programmes électoraux des partis politiques}

L'offre politique des partis marocains se trouve influencée par leurs histoires, mais également par leur force de proposition en fonction de leurs stades d'évolution. Ainsi, des partis qui ont cumulé des expériences dans la gestion (gouvernement), ceux qui ont eu des expériences dans l'opposition, ou alors ceux qui ont cumulé des expériences dans le travail de terrain, n'ont pas trouvé de difficulté à cristalliser ce qu'ils pensent être en adéquation avec les attentes des citoyens.

Toutefois, ce qui détermine réellement la capacité d'un parti à proposer des programmes en adéquation avec les attentes de la population est le degré d'adhésion des individus au travail politique, et il semble que nous n'avons pas encore atteint ce haut niveau d'adhésion où les individus peuvent se refléter dans l'offre des programmes politiques.

Les programmes électoraux des partis politiques au Maroc ont été basés sur des statistiques globales obtenues des sources officielles (e.g., HautCommissariat au Plan, rapports internationaux, départements ministériels, bilan du gouvernement sortant, etc.), ce qui a eu pour résultat des similitudes importantes des bilans dressés et des objectifs fixés (inspirés des politiques déjà lancées et/ou des initiatives royales) avec quelques différences près de chiffres. En effet, la compréhension effective des besoins de l'électeur dans l'optique d'influencer sa décision de vote exige le recours à des enquêtes spécifiques d'avis et d'opinion publique, ce qui n'est pas à confondre avec les sondages qui sont élaborés par la presse ou certains organismes spécialisés à destination du grand public. Ainsi, le principal pilier du Marketing politique se trouve réduit à une simple aspiration de vente d'hommes politiques.

\subsection{Le marketing stratégique : le maillon faible du marketing politique au Maroc}

Les partis évoluent généralement, et ce quel que soit le système politique, dans un environnement incertain et complexe. Ainsi, le changement 
est une caractéristique commune à tous les partis et toutes les organisations des partis politiques, et c'est une caractéristique qui marque également leur environnement extérieur.

Les attentes de l'électorat dans son ensemble, ou de certaines de ses franges, évoluent. D'autre part, les difficultés économiques, ainsi que les enjeux politiques nationaux et internationaux mettent continuellement les partis politiques face à de nouvelles responsabilités et de nouveaux défis.

Ces changements d'environnement et ces nouveaux enjeux peuvent renforcer ou affaiblir un parti politique, contribuer à la réalisation de ses objectifs, ou alors avoir une incidence négative ou compromettre son développement. Pour réussir dans ce type d'environnement, les partis politiques sont appelés à faire preuve de concentration, de détermination et disposer de capacités institutionnelles d'adaptation. Il est crucial pour un parti politique d'avoir une vision commune de sa nature, de ses objectifs et des actions à mettre en œuvre pour les atteindre.

Pour ce faire, la planification stratégique devient une démarche fondamentale pour permettre aux partis politiques de forger une vision commune et partagée, ou alors pour édifier un projet communautaire cohérent. La planification stratégique permet ainsi aux partis politiques de prendre suffisamment de recul par rapport à leurs activités et préoccupations quotidiennes, et de réfléchir à des questions plus fondamentales et à long terme. Elle leur permet également de définir des objectifs réalistes visant à rétablir, maintenir ou à accroître leur force institutionnelle. L'instauration d'un processus de planification stratégique par les partis politiques est susceptible de stimuler les débats internes et de favoriser l'émergence d'idées, tout en unissant les membres et adhérents autour d'objectifs communs. Ceci permet, par ailleurs, de définir le positionnement politique, de formuler des stratégies d'avenir et de définir des repères de progression du parti.

L'une des caractéristiques marquantes de la vie politique au Maroc, comme dans nombreux autres pays, c'est l'existence d'une tension constante entre la nécessité de répondre à l'électorat dans l'immédiat, et la formulation d'objectifs et de stratégies à long terme.

Comme réaction à ce contexte, les partis politiques marocains sont le plus souvent contraints de concentrer leurs efforts sur des crises socioéconomiques d'actualité, à court terme, au détriment du développement de véritables stratégies à moyen et long terme, et de l'organisation interne nécessaire à ces stratégies. Ceci se traduit par une diminution de la confiance accordée par le public aux partis politiques en tant qu'acteurs de représentation et de gouvernance, surtout dans le cas des démocraties émergentes.

Dans le contexte marocain, les pratiques de ciblage des partis politiques marocains ne semblent pas aussi faciles à identifier. Nous retrouvons ainsi des partis politiques qui ciblent des catégories spécifiques, 
comme le cas du PJD en l'occurrence. En effet, le religieux pèse toujours sur le discours politique du PJD avec une forte capacité de dissimulation en fonction des contextes.

Le parti avance l'argument du discours démocratique qui croit dans l'état civil, mais dès qu'il y ait intensification dans la concurrence électorale, le parti refait référence au texte coranique et la Sunna du Prophète, et on recommence à parler de la nature religieuse du pays, alors que le reste des partis politiques n'ont pas encore atteint un tel niveau MP\#3.

Le PJD semble bien savoir exploiter le côté émotionnel et spirituel, aussi bien de ses adhérents que de sa cible, en mettant en avant le discours de « se considérer tous comme des frères dans la religion, rassemblés autour de Dieu et s'aiment en Dieu ». Ce discours trouve une forte résonance dans la communauté, car elle est psychiquement préparée pour accepter un tel discours.

\section{Politique de communication et définition du produit « candidat » des partis marocains}

\subsection{Politique de communication}

$\mathrm{Au}$ Maroc, il est presque évident que la communication politique a totalement changé de dimension. Après avoir été quasi-confidentielle, balbutiante et monopolisée par certaines instituions, la communication politique devient une pratique réclamée par l'ensemble des acteurs politiques. Les partis politiques ont pris peu à peu conscience des riches possibilités techniques et des multiples avantages en termes de valorisation de l'image publique, ainsi qu'en termes de construction d'un discours politique efficace que les médias offraient. Ils s'en sont donc saisis pour les intégrer aux diverses stratégies de communication déjà mises en place par les politiciens, leurs conseillers en communication et leurs équipes de campagne.

Les partis et candidats ont utilisé une variété de moyens pour communiquer avec les citoyens. Si les partis ont largement profité de l'ouverture médiatique et de la libéralisation de l'espace audiovisuel, l'usage des canaux de communication interactifs par les candidats continue à primer sur l'usage des autres nouveaux moyens de communication.

L'outil qui a été le plus utilisé dans les élections est le discours oral. En fait, le contexte général de rejet de la lecture fait que l'oral ait grandement affecté l'électeur que l'écrit. D'autre part, le constat est que ceux qui lisent les programmes électoraux ne sont pas forcément des gens qui votent.

Le niveau d'instruction réduit chez une frange importante de l'électorat (entre autres l'analphabétisme) conduit à une incompréhension des programmes et donc au vote axé plutôt sur la personne ou sur le logo du parti. Nous sommes alors plus proches du modèle propagandiste que du modèle du marketing politique. 
Notre interviewé souligne que les citoyens qui ont un certain niveau d'études et qui sont capables de lire les programmes électoraux des partis enregistrent des taux de participation très faibles dans les élections, et donc n'influencent pas les résultats finaux de celles-ci :

Ce sont des gens qui ont bien compris la politique et le jeu électoral, et ils savent très bien que les grandes décisions économiques et de sécurité dépassent le gouvernement. Ils ne croient pas à la capacité des parlementaires marocains à produire de bons textes juridiques, et savent très bien que le gouvernement est incapable de prendre d'importantes décisions exécutives. Toutefois, la plupart des citoyens sont influencés par le discours oral MP\#5. Par ailleurs, sur le plan de la stratégie de communication, et à l'exception de quelques grands partis suffisamment médiatisés qui font recours à des spécialistes en la matière, certains observateurs relèvent l'existence de dissemblances flagrantes dans les discours de candidats appartenant au même parti, d'une part, et d'autre part le manque de coordination et de communication interne entre le sommet du parti et les dirigeants locaux.

Au registre de la communication, les partis utilisent des slogans courts en arabe ou en dialecte «Darija » comme axe central de communication. Par ailleurs, et pour ce qui est des canaux utilisés, il devient quand même clair que l'âge des affiches sur les murs comme seul outil de communication semble révolu. On assiste alors à un renforcement d'autres outils et canaux marketing média ou hors média, comme les tournées organisées, la distribution de prospectus électoraux, les meetings, l'usage de la télévision (interventions des représentants dans les journaux télévisés, émissions pour leaders et spots publicitaires), le passage dans les stations radio, le recours à la presse écrite y compris celle indépendante, ou alors l'organisation d'événements publics ou privés, voir même des réceptions ou des « festins » surtout dans le rural. La nouveauté introduite lors des élections 2016 est sans doute l'emploi des technologies d'information et de communication et du marketing direct (réseaux sociaux, informations dynamiques sur les sites, envois de SMS, emailings, forums discussion, programmes téléchargeables, etc.).

Les partis ont été conscients de l'importance de la mise en place d'une infrastructure opérationnelle pour garantir l'efficacité des campagnes électorales pour les élections de 2016. Certains partis organisés ont ainsi fait appel à leurs fonctions internes (cellules / départements / pôles de communication) pour mettre en place des plans de campagne. La composition de ces départements variait d'un parti à un autre. Dans la majorité des cas, des directeurs de campagne ont été désignés pour orchestrer les actions et les réalisations. Outre les directeurs de campagne au niveau national, des comités de coordination ont été désignés et comprenaient des responsables de la logistique, des finances, relations presse, veille ou surveillance, et dans certains cas on pouvait trouver même des conseillers. Presque la totalité des 
partis s'est contentée des ressources internes, le recours aux agences s'est fait principalement pour la réalisation ou la production de capsules ou autres contenus destinés aux médias.

Dans des phases préalables aux législatives, certains partis bien organisés dans leurs campagnes ont mis en place des infrastructures nécessaires et ont procédé à une répartition des tâches tout en identifiant des équipes de soutien ; il s'agit notamment des partis PJD et du PPS.

\subsection{Le choix des candidats}

Certains observateurs considèrent que le PJD est le seul parti qui a fait preuve d'une certaine rigueur dans la nomination de leurs candidats, et ce au niveau national. Toutefois, pour le PJD il y a eu des exceptions organisationnelles à la procédure de nomination puisque le secrétariat général du PJD peut recourir à la nomination sans restriction pour les autres partis, on assiste à un respect des procédures de nomination dans certains arrondissements, tout en sélectionnant les politiciens les plus expérimentés.

Il est à noter que le respect de la procédure de nomination est considéré parmi les éléments qui donnent une bonne image au parti, en ce sens que cela démontre que l'on est face à parti démocratique.

\section{Enjeux et perspectives du E-Marketing au Maroc}

\subsection{Les chiffres clés de la révolution numérique au Maroc}

Les résultats de l'enquête annuelle sur l'équipement et l'usage des technologies de l'information par les Marocains réalisée par L'Agence Nationale de Réglementation des Télécommunications (ANRT) en 2016 montre que la transformation digitale de la société marocaine s'opère de façon progressive. Cela dit les nouvelles technologies de communication ont pu se trouver une place même dans le rural. Ainsi, la téléphonie mobile s'est généralisée pour la quasi-totalité des ménages dans le milieu rural. Cela confirme la démocratisation de ce service. Ainsi, $90,7 \%$ des individus âgés de 12 à 65 ans en sont équipés en milieu rural. Selon la même enquête, $67 \%$ des individus âgés de 12 à 65 ans, équipés en téléphone mobile, disposeraient en 2016 d'un smartphone, contre $54,7 \%$ une année auparavant. L'adoption de cet outil, fortement utilisé pour accéder à Internet (par 93\% des individus qui en sont équipés), montre l'intérêt des utilisateurs. $68,5 \%$ des ménages seraient actuellement équipés d'un accès à Internet et $66,5 \%$ des ménages équipés le sont avec la technologie mobile.

Selon les données de l'Agence nationale de réglementation des télécommunications (ANRT), le Maroc compterait 18,5 millions d'internautes, passant de 57,1\% en 2015 à $58,3 \%$ en 2016. Avec une progression impressionnante dans le monde rural (de 42,2\% en 2015 à 44,4\% en 2016). Le nombre d'internautes, même s'il reste important, montre 
l'existence encore d'un potentiel significatif, pouvant impacter positivement la pénétration de l'Internet et des accès, les taux d'utilisation ainsi que les types d'usage sur Internet. Les réseaux sociaux, la presse, l'actualité, les sites de jeux, loisirs et de sport, représentent les contenus favoris des internautes avec des taux pouvant atteindre $80,7 \%$.

\subsection{Les réseaux sociaux, principale activité en ligne des Marocains}

En ce qui concerne les réseaux sociaux, les Marocains continuent à y enregistrer une présence forte. Concernant le contexte des législatives de 2016, le rapport publié la même année par MEDIANET LABS sur le comportement de l'internaute africain sur le web et sur l'utilisation de Facebook en Afrique précise que 13 millions de Marocains utilisent Facebook, soit 39\% de la population, avec une évolution de 20\% par rapport à 2015.

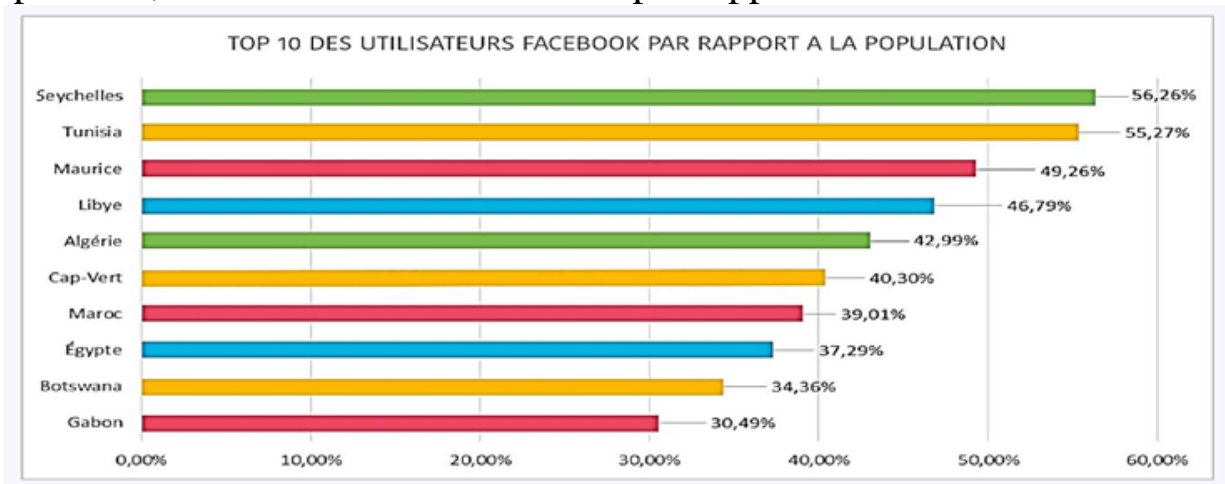

Figure 1. TOP 10 des utilisateurs Facebook par rapport à la population

Le Maroc est l'un des plus gros utilisateurs du Facebook, derrière l'Afrique du Sud, le Nigeria, l'Algérie et l'Égypte. Cependant avec ses 39\% d'utilisateurs, le Maroc reste loin derrière ses voisins du point de vue du nombre d'utilisateurs en pourcentage de la population. Ainsi $42,99 \%$ des Algériens ont un compte Facebook et en Tunisie plus de la moitié de la population est connectée au réseau.

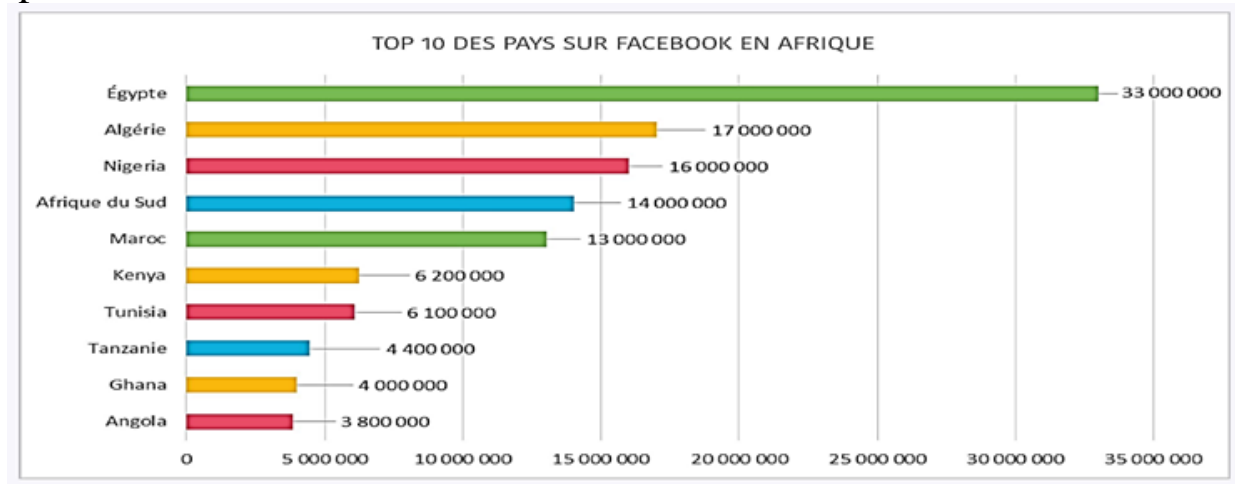

Figure 2. TOP 10 des pays sur Facebook en Afrique 
En 2018, le rapport sur la digitalisation au Maroc publié par Hootsuite, montre que le nombre d'utilisateurs d'Internet par les Marocains s'élève à 22.5 millions de citoyens, soit $36 \%$ de la population totale.

D'autre part, l'usage des médias sociaux concerne 16 millions de marocains, soit une évolution de plus de 3 millions d'utilisateurs par rapport à l'année 2015. Ceci démontre que les Marocains sont de plus en plus connectés, et sont très actifs sur les médias sociaux. Ces chiffres montrent également que la majorité des classes sociales utilisent les médias sociaux, et que l'information peut être disponible pour la grande majorité des citoyens.

La même étude montre que $96 \%$ des Marocains sont aujourd'hui dotés de téléphone mobile, et que $57 \%$ ont des smartphones. L'étude montre également que le temps moyen de connexion à Internet par jour est de $2 \mathrm{~h} 53$ minutes, et que le temps passé à utiliser les médias sociaux est de $2 \mathrm{~h} 24$ minutes. Ceci montre clairement que les Marocains passent le plus clair de leur temps de connexion à utiliser les médias sociaux.

Pour ce qui est des médias sociaux, on remarque que WhatsApp est la plateforme la plus utilisée par les Marocains, avec un taux d'usage de $53 \%$, et qui dépasse Facebook qui vient en deuxième position avec 50\%, et YouTube qui vient en troisième position $(41 \%)$.

\subsection{Présence « réseaux sociaux » des partis politiques marocains}

Les campagnes de sponsoring en masse des pages Facebook ont provoqué une augmentation importante du nombre d'abonnés aux pages des personnalités politiques. Sur ce volet, deux champions nationaux sont identifiés : le Parti de la Justice et du Développement (PJD) connu pour son « armée digitale » redoutable avec près de 450.000 abonnés et le Parti Authenticité et Modernité (PAM) avec presque 180.000 abonnés.

Le PJD est sans doute le parti le plus présent dans les sites de réseautage social, et il possède une grande capacité de mobilisation à travers ces sites web. Il est aussi l'un des premiers partis politiques qui a su pénétrer les sites de réseautage social et le monde virtuel numérique. Le PJD semble avoir une bonne longueur d'avance sur les autres partis sur le monde virtuel. Le parti est en tête de classement avec plus de 1 million de fans sur Facebook, en plus de 80.000 followers sur Twitter. Le PAM, quant à lui, a avec plus de 500.000 fans sur Facebook, suivi par le PPS avec 358.000 fans. Le reste des partis nationaux sont loin derrière avec quelques dizaines de milliers de fans. Pour ce qui est des partis progressistes et de gauche tels que l'Union Socialiste des Forces Populaires (USFP), ils sous-estimaient le potentiel des sites de réseautage social, et considéraient les utilisateurs de ces sites web comme étant moins conscients et plus influençables, voir même la remise en cause de la véracité des profils des utilisateurs de ces sites web. 
La particularité de la société, sa diversité et les particularités des régions font que, malgré un taux de pénétration d'Internet de $42,78 \%$ fin mars 2016 , et qui est à $63 \%$ en janvier 2018, l'essentiel de la campagne électorale se réalisait sur le terrain réel. D'une manière générale, les partis politiques marocains restent très attachés aux outils traditionnels et classiques, à savoir l'interaction directe, les rencontres dans les maisons, les souks, les douars, à la force d'animation, de musique populaire, de distribution massive de flyers, etc. Pour ce qui est de la communication politique sur les réseaux sociaux, les partis l'ont déjà expérimentée lors des communales et régionales précédant les législatives, à des échelles différentes. Leur nouveau canal, qualifié de « cheval de bataille » semble être la web tv et la web radio.

Le rival le plus redoutable du PJD sur la scène politique, l'est aussi sur Internet. Le Parti Authenticité et Modernité (PAM) semble être un fort rival. Fort de ses nouveaux services web : PAM Tv et PAM Radio, le parti dit du tracteur, puisqu'il adopte le tracteur comme logo, possède un pôle numérique bien structuré qui confère un caractère institutionnel à sa présence sur la toile.

En 2016, tout comme le PAM, le Parti de l'Istiqlal (PI), le Parti du Progrès et du Socialisme (PPS), le Mouvement Populaire (MP), le Rassemblement National des Indépendants (RNI) et l'Union Socialiste des Forces Populaires (USFP) ont réalisé un développement remarquable en termes de représentativité sur les réseaux sociaux. Néanmoins, ces formations, notamment le PPS, un des pionniers de l'utilisation de Facebook, enregistrent une forte activité durant les échéances électorales, mais avec une chute spectaculaire d'activité hors élections.

Le point commun de ces partis, c'est qu'ils ont tous repensé leurs plateformes web pour donner plus d'attractivité et un meilleur rendement, comme ils ont lancé de nouveaux services d'information et de communication.

Ces percées peuvent être expliquées par une prise de conscience du rôle déterminant que peuvent jouer les réseaux sociaux dans la consolidation de l'e-réputation et de l'influence virtuelle des partis sur un électorat de plus en plus important et qui pourrait peser dans la balance le jour du scrutin.

Les autres partis ne sont pas en reste. Mais si certains sont appelés à compenser leur part de présence dans les médias publics, il est clair que, paradoxalement, les "grandes" formations lui ont damé le pion sur ce front aussi. Certaines formations sont même inaudibles sur les réseaux sociaux, une erreur fatale et incompréhensible, en ce temps où le virtuel est devenu un véritable vivier électoral.

\section{Conclusion}

Le marketing politique est un concept théorique universel, la compréhension de sa démarche stratégique et des réalités de sa pratique sur un contexte empirique diffèrent tel que le contexte Maroc n'est pas un simple 
exercice de transposition, elle nécessite un effort d'adaptation, compte tenu des différents facteurs propres au contexte en question, les différentes perceptions des acteurs (Makry, 2019), la grande limitation des moyens financiers des partis politiques marocains, le faible intérêt que porte l'élite partisane à la professionnalisation de la communication, la concentration des efforts politique sur des crises socio-économiques d'actualité à court terme, au détriment du développement de véritables stratégies à moyen et long terme, ainsi que la forte évolution de la connectivité des marocains.

L'enjeu de chaque parti politique est d'améliorer sa performance électorale, acquérir une majorité de vote pour pouvoir gouverner. Le Marketing politique se présente comme possibilité pour les décideurs politiques. Dans cette contribution nous avons choisis d'étudier le contexte des élections législatives 2016 pour savoir si le marketing politique présente des caractéristiques similaires dans des contextes politique et socioéconomique différents. L'objectif étant de pouvoir contextualiser notre étude empirique principale qui tente de savoir comment l'utilisation des outils Marketing Politique peut améliorer la performance électorale des Partis Politiques Marocains ? en interrogeant cette fois-ci les praticiens et les leaders politiques.

Cette étude de contextualisation, nous a permis d'affiner le cadre théorico-conceptuel de notre étude et d'orienter plus précisément la démarche sur le terrain. Nous avons ainsi pu délimiter la collecte des données par rapport à l'objet étudié et son contexte. Nous avons également retenu des éléments évoqués par ces experts, auxquels nous n'avions pas pensé auparavant.

Les résultats de cette étude de recherche contextualisée feront objet de prochaine publication

\section{References:}

1. Beji, I. (2016). Chiffres clés sur les réseaux sociaux en Afrique: Facebook, Linkedin, Instagram) présentés à l'African Digital Summit 2016, MEDIANET

2. Bru, M. (1991). Les variations didactiques dans l'organisation des conditions d'apprentissage. Toulouse. Editions Universitaires du SUD.

3. Buchanan, DA. \& Bryman, A. (2007). Contextualizing Methods Choice in Organizational Research, Academy of Management Review.

4. Dey, A. (2001). Understanding and Using Context. Personal Ubi Comp 5,

5. Egan, J. (1999). «Political marketing: Lessons from the mainstream », Journal of Marketing Management. 
6. Guba, EG. \& Lincoln, Y-S. (1994). Competing Paradigms in Qualitative Research, Handbook of qualitative research, 1994.

7. Kotler, P. (1975). "Overview of political candidate marketing », Advances in Consumer Research.

8. Makry, H. et al. (2019). Political Marketing in Morocco: From Theories to Stereotypes, Definition and Comparison, International Review of Management and Marketing, 2019, 9(4), 80-86.

9. Makry, H. et al. (2020). Comprendre Le Marketing Politique: Théorie, Concept et Stratégie Understanding Political Marketing : Theory, Concept and Strategy, Revue Internationale des Sciences de Gestion, Volume 3 : Numéro 3

10. Marcel, JF. (2004). Le concept de contextualisation : un instrument pour l'étude des pratiques enseignantes, Revue Française de pédagogie

11. Pourtois, J.P. \& Desmet, H. (2004). L'éducation implicite. Paris : Presses Universitaires de France.

12. Shama, A. (1975). "Applications of marketing concepts to candidate marketing », Advances in Consumer Research.

13. Parrini-Alemanno, S. (2007). Contexte et contextualisation dans l'approche qualitative de la communication organisationnelle RECHERCHES QUALITATIVES - Hors-Série - numéro 3.

\section{Annexe 1}

Rapport annuel de l'ANRT 2016 :

https://www.anrt.ma/sites/default/files/rapportannuel/rapport_annuel20

16 anrt vf 2.pdf

Rapport sur la digitalisation au Maroc Hootsuite 2018 :

https://fr.slideshare.net/EveryLeader/digital-in-morocco 